\title{
Development of a mid-sized Schwarzschild-Couder telescope for the Cherenkov Telescope Array
}

\author{
Robert A. Cameron ${ }^{\text {* }}$ \\ on behalf of the Cherenkov Telescope Array Consortium \\ ${ }^{a}$ SLAC National Accelerator Laboratory, 2575 Sand Hill Road, Menlo Park, CA, USA 94025
}

\begin{abstract}
The Cherenkov Telescope Array (CTA) is a ground-based observatory for very high-energy (10 GeV to $100 \mathrm{TeV}$ ) gamma rays, planned for operation starting in 2018. It will be an array of dozens of optical telescopes, known as Atmospheric Cherenkov Telescopes (ACTs), of $8 \mathrm{~m}$ to $24 \mathrm{~m}$ diameter, deployed over an area of more than 1 square $\mathrm{km}$, to detect flashes of Cherenkov light from showers initiated in the Earth's atmosphere by gamma rays. CTA will have improved angular resolution, a wider energy range, larger fields of view and an order of magnitude improvement in sensitivity over current ACT arrays such as H.E.S.S., MAGIC and VERITAS.

Several institutions have proposed a research and development program to eventually contribute 36 medium-sized telescopes ( $9 \mathrm{~m}$ to $12 \mathrm{~m}$ diameter) to CTA to enhance and optimize its science performance. The program aims to construct a prototype of an innovative, Schwarzschild-Couder telescope (SCT) design that will allow much smaller and less expensive cameras and much larger fields of view than conventional Davies-Cotton designs, and will also include design and testing of camera electronics for the necessary advances in performance, reliability and cost. We report on the progress of the mid-sized SCT development program.
\end{abstract}

Keywords: gamma ray, Schwarzschild-Couder telescope, Cherenkov Telescope Array

\section{INTRODUCTION}

The Cherenkov Telescope $\operatorname{Array}^{1}$ (CTA) Consortium is undertaking a large international effort to design and build the world's most sensitive gamma-ray observatory to operate in the tens of $\mathrm{GeV}$ to $100 \mathrm{TeV}$ energy range. The effort is motivated by the recent successes of VERITAS in the USA and H.E.S.S. and MAGIC in Europe, which have collectively provided a glimpse of the great scientific potential for particle physics and astrophysics offered by the gamma-ray sky at very high energies. CTA is included in the 2008 roadmap of the European Strategy Forum on Research Infrastructures (ESFRI) where it is listed as one of the Magnificent Seven projects comprising the European strategy for astroparticle physics published by ASPERA. CTA is also highly ranked in the strategic plan for European astronomy of ASTRONET. In the USA, the Astro2010 Decadal Survey strongly endorsed the scientific promise of a next-generation atmospheric Cherenkov telescope array and recommended significant USA participation in CTA. Consequently, in May 2010 the USA groups formerly comprising the Advanced Gamma-ray Imaging System (AGIS) Collaboration joined the CTA Consortium.

CTA is an imaging atmospheric Cherenkov telescope (IACT) array, which will detect gamma rays by imaging the Cherenkov light of electromagnetic showers in the atmosphere, a technique that can reach its full potential only with an array of telescopes of the scale of CTA. The CTA conceptual design incorporates telescopes of three different sizes, denoted small-, medium-, and large-sized telescopes (SST, MST, and LST). This array is planned to provide wide spectral coverage, imaging capabilities with arcminute accuracy, and sky- survey capabilities. CTA is planned to provide full-sky access through installations at northern and southern sites. The southern array will capitalize on extension of the accessible energy range to well below $100 \mathrm{GeV}$ and to above $100 \mathrm{TeV}$, whereas the northern array will consist of the low-energy and mid-energy instrumentation (from a few tens of GeV to $10 \mathrm{TeV}$, provided by the MSTs and LSTs). The latter array will be dedicated mainly to northern extragalactic objects, which at cosmological distances are not expected to have significant detectable emission above $10 \mathrm{TeV}$ because of gamma-ray absorption by the extragalactic background light. Some of the observation time will be open to the general community, with provided user support facilities.

*rac@slac.stanford.edu

Updated 1 March 2012

Presented at the SPIE Astronomical Telescopes+ Instrumentation Conference Amsterdam, The Netherlands, July 1-6, 2012

Work supported by US Department of Energy contract DE-AC02-76SF00515. 
The current vision for CTA calls for the southern array including about 60 medium-sized telescopes (MSTs), which primarily cover the core energy range of $100 \mathrm{GeV}-10 \mathrm{TeV}$, to be built in two phases. Construction of the first phase of about 23 telescopes would ideally commence at the conclusion of the CTA Preparatory Phase (expected to end in 2014) and be funded from European sources. The USA groups in the CTA Consortium have proposed a second-phase construction of 36 telescopes, to begin roughly two years later, bringing the MST array to its full scientific potential. Each construction phase would take 4-5 years and partially overlap in time. The first phase of MST construction is planned to use a single-mirror Davies-Cotton (DC) telescope design. The additional time prior to the second phase of construction is being used to investigate and develop the novel two-mirror Schwarzschild-Couder (SC) design, which has the potential to deliver better performing (or more cost-effective) telescopes. The SC telescope configuration is also being considered for the small-sized telescopes in CTA.

In the USA, the current proposals to the National Science Foundation and the Department of Energy are to fund a research and development program to design, construct and test a prototype mid-sized SC telescope (SCT), and so to accurately understand the performance and cost of such a telescope design in order to obtain the maximum performance from the final MST array envisioned for CTA. Following the construction and test of the prototype SCT, the next step would be a funded project to construct and install 36 SC telescopes with the CTA array, on a timescale lagging the start of the European-led Construction Phase for CTA.

The remaining sections of this paper outline the main motivations for and the benefits of the SCT design, and provide more detail on design efforts underway for the telescope mechanical structure, the telescope optics, and the imaging camera, plus planned testing, and a summary of the planned work.

\section{MOTIVATION FOR A MEDIUM-SIZED SC TELESCOPE FOR CTA}

The principal motivations for a new gamma-ray telescope design are the need for better angular resolution and wider field of view. Better angular resolution translates directly into better sensitivity to point sources, which are generally studied in the regime limited by the background from charged cosmic rays. It also enables mapping spatially extended sources in sharper detail: resolving structure in such sources is important to understanding the particle acceleration processes involved. A wider field of view also allows better measurements of extended sources, while at the same time improving the efficiency of survey observations and the response to gamma-ray bursts, and increasing the effective collection area of the telescope by enabling it to detect showers at larger impact parameter.

The angular resolution of current IACT arrays is still far from the intrinsic limit determined by fluctuations in electromagnetic shower development. The angular resolution can be substantially improved for large arrays of IACTs if the camera pixels can be reduced below the $\sim 0.15$ degree size in current-generation IACTs and the image quality of the telescope optical system (which is matched to the pixel size for cost optimization) can be correspondingly improved. Analysis of simulated Cherenkov images shows the utility of a higher-resolution system. To fully utilize the information in the shower image, the pixel resolution should match or oversample the transverse size of the central region of the cascade, typically spanning a few minutes of arc. Figure 1 illustrates a $40 \%$ sensitivity improvement for an array consisting of telescopes with the SCT performance, which corresponds to a factor of two reduction in observation time for background-limited sources. Depending on the spatial distribution of weak sources (galactic or extragalactic) this could result in an increase in the CTA source catalog by $40-70 \%$.

Improving the camera resolution while at the same time increasing the field of view is problematic for existing IACT designs. A telescope with prime focus optics has comatic aberrations, which can be reduced only by increasing the focal length. This in turn increases the plate scale and therefore the physical size of the individual pixels of a given angular size in the camera. The cost of the resulting telescope is dominated by the cost of thousands of expensive large-aperture photomultiplier tubes (PMTs). For example, the design of a conventional telescope of this type for CTA has a $2.5 \mathrm{~m}$ diameter camera with approximately 2000 channels (pixels) in an 8-degree diameter field of view, where each channel has an angular diameter of 0.18 degrees, which corresponds to $5 \mathrm{~cm}$ on the focal plane. 

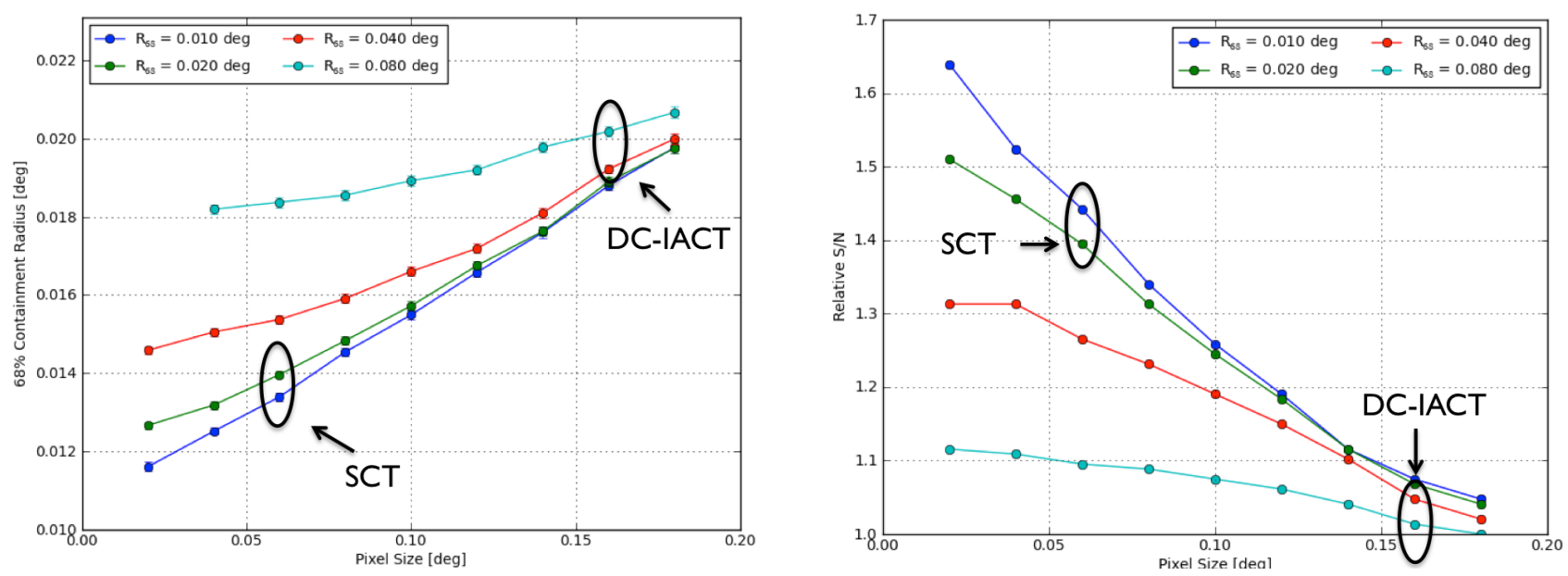

Figure 1. Simulated effect of the camera pixel size on gamma-ray angular resolution (left, $1 \mathrm{TeV}$ gamma-rays) and signalto-noise ratio (right, $316 \mathrm{GeV}$ gamma-rays) for a 61-telescope mid-sized array as envisioned for CTA, using telescopes of either the SCT design or conventional Davies-Cotton IACTs, as a function of the camera pixel size. The curves correspond to different values for the $68 \%$ containment radius for the point-spread function (PSF) of the optical system. The smaller optical PSF of the SCT design justifies the investment in smaller pixels in the camera, with both working together to yield $40 \%$ better overall performance. The actual SCT array performance will be bracketed by the green and blue lines; the performance of the conventional telescope array will be between the red and cyan lines.

To overcome such limitations, we are planning to implement a promising new approach to IACT design, by constructing a two-mirror Schwarzschild-Couder telescope ${ }^{2,3}$. This telescope design was first proposed in 1905 by Karl Schwarzschild ${ }^{4}$, who provided analytic solutions (Schwarzschild aplanats) for the figures of both mirrors, which correct for both spherical and comatic aberrations near the optical axis. Only recently have aspheric mirror fabrication technologies reached the sophistication and cost needed to build a telescope of this type. In the proposed design, we have also optimized the two aspheric mirrors and slight focal plane curvature to minimize astigmatism (Figure 3). A critical benefit of this design is the small plate scale, because the secondary mirror de-magnifies the image and considerably reduces the focal length. This property makes the telescope compatible with small-sized, integrated photosensors, such as multi-anode PMTs (MAPMT) and silicon photomultipliers (SiPM), thus dramatically reducing the costs per pixel from several hundred dollars for a large aperture PMT to several tens of dollars. The SCT is by design a wide field of view instrument, and in addition the two-mirror aplanatic design does not introduce wavefront distortions (cf. the VERITAS Davies-Cotton telescopes have a $\sim 4$ ns time spread), allowing tighter trigger coincidence requirements and a lower energy threshold.

The relatively small pixel size and wide field of view of the camera, driven by the science requirements, results in a high channel count and density, for which custom ASIC read-out electronics are a natural solution. The intrinsic time structure of the Cherenkov light pulse and the benefit of using signals from multiple telescopes at the trigger level argue for waveform sampling with a frequency of $\sim 1 \mathrm{GHz}$ and a buffer depth of at least $8 \mu$ seconds, respectively. A high degree of integration in the camera is also beneficial from the standpoint of reliability and maintenance. The TARGET ASIC meeting these camera requirements is described in more detail in Section 5.

\section{TELESCOPE MECHANICAL STRUCTURE}

The prototype SCT will use an innovative altitude-azimuth telescope structural design produced at the Institute of Nuclear Physics of the Polish Academy of Sciences (IFJ PAN) in Krakow, shown in Figure 2. A rigid central cone in the shadow of the secondary mirror supports both the camera and the secondary mirror.

The design and development of the mechanical structure for the SCT will be coordinated through CTA Collaboration members at the Argonne National Laboratory (ANL) in Illinois. ANL has extensive experience in mechanical design for large scientific instruments, and will be assisted by participants from IFJ PAN in Krakow Poland, DESY-Zeuthen in 
Germany, and collaboration members at UC-Los Angeles (UCLA), Columbia University, Barnard College, and the Smithsonian Astrophysical Observatory (SAO) in the USA. Engineers with the IFJ PAN-Krakow and DESY-Zeuthen groups will be involved in all mechanical and drive design activities, supported by their correspondent institutions.

The design of optical support structure (OSS) facilitates maintaining the critical alignment between the secondary mirror and the camera, while at the same time minimizing stresses on the primary mirror. To achieve and maintain the required optical point-spread function (PSF), the SCT has more stringent requirements on the positioning and alignment of the two mirrors than a conventional Davies-Cotton (DC) IACT. Assessment of the expected achievable deformations and deflections of the mechanical structure indicates that the optical surfaces must be positioned with an accuracy better than a few $\mathrm{mm}$, be aligned to better than 0.2 milliradians, and not deviate from a perfect aspheric surface by much more than $0.1 \mathrm{~mm}$ over a length of $1 \mathrm{~m}$.
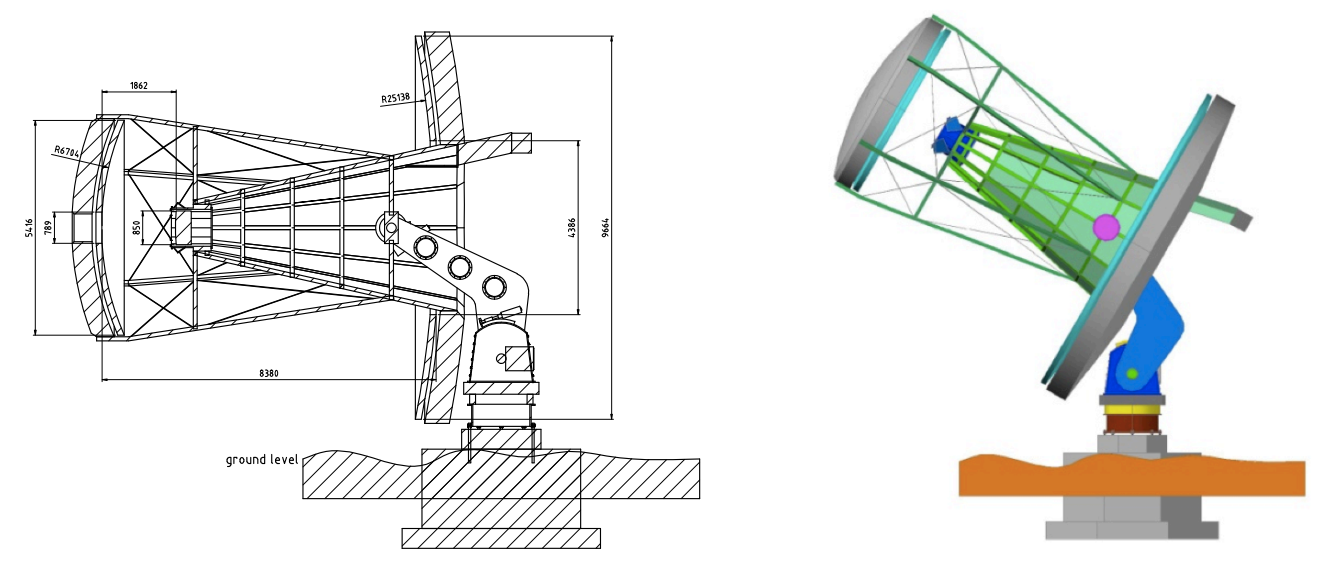

Figure 2. The IFJ PAN-Krakow design for the SCT structure, using a rigid central cone for mirror support. The dimensions shown on the left figure are in $\mathrm{mm}$.

The DESY-Zeuthen group has led the design and prototype fabrication of a mount for the medium-sized Davies-Cotton telescopes of CTA. The azimuth and elevation motors used for those telescopes also will be used for the SC telescope mount, and will be provided by the DESY-Zeuthen group for use in the prototype SCT. DESY-Zeuthen also will provide the electrical system for the prototype SCT, plus controllers for the azimuth and elevation drives, associated software, and will develop the safety system for the prototype telescope. The telescope mount will be designed by the IFJ PANKrakow group to mate to an existing foundation at the Fred L. Whipple Observatory (FLWO), managed by SAO, adjacent to the existing VERITAS array at that site. After assembly and local testing of the telescope mount and associated electrical systems at IJF PAN, the components will be shipped directly to FLWO. Under ANL oversight, the OSS will be fabricated and assembled and test fit at the manufacturer facility, then disassembled and shipped to FLWO.

\section{TELESCOPE OPTICS}

The optical system of the telescope consists of a $9.5 \mathrm{~m}$ aperture primary mirror with $4.4 \mathrm{~m}$ central hole, a $5.4 \mathrm{~m}$ demagnifying secondary mirror, and an $0.8 \mathrm{~m}$ diameter, 8 degree field-of-view focal plane (Figure 3). It has $5.6 \mathrm{~m}$ focal length and is derived from the exact Schwarzschild aplanatic solution ${ }^{4}$. To achieve a wide field of view and high imaging resolution, the on-axis spherical and comatic aberrations are exactly corrected, and the focal plane is curved optimally to minimize astigmatism. Extensive simulations have shown that this solution is close to optimal for ground-based gammaray observations. The point-spread function is less than 4 arcminutes for field angles within 4 degrees of the optical axis.

The aspheric primary and secondary mirrors are segmented, each consisting of three types of petal panels, in concentric rings (Figure 3). Reducing the size of individual panels increases the assembly and alignment costs, which scale with the number of panels, while increasing the size of individual panels significantly beyond $1.3 \mathrm{~m}$ (diagonal) dramatically 
increases fabrication costs. A challenge of the SCT design is its aspheric mirrors and in particular the significant curvature of the secondary. Detailed telescope simulations indicate that the specifications for the figures of the primary and secondary mirrors are typical of those for sub-mm radio telescopes ( 100 $\mu \mathrm{m}$ tolerances), since IACTs focus
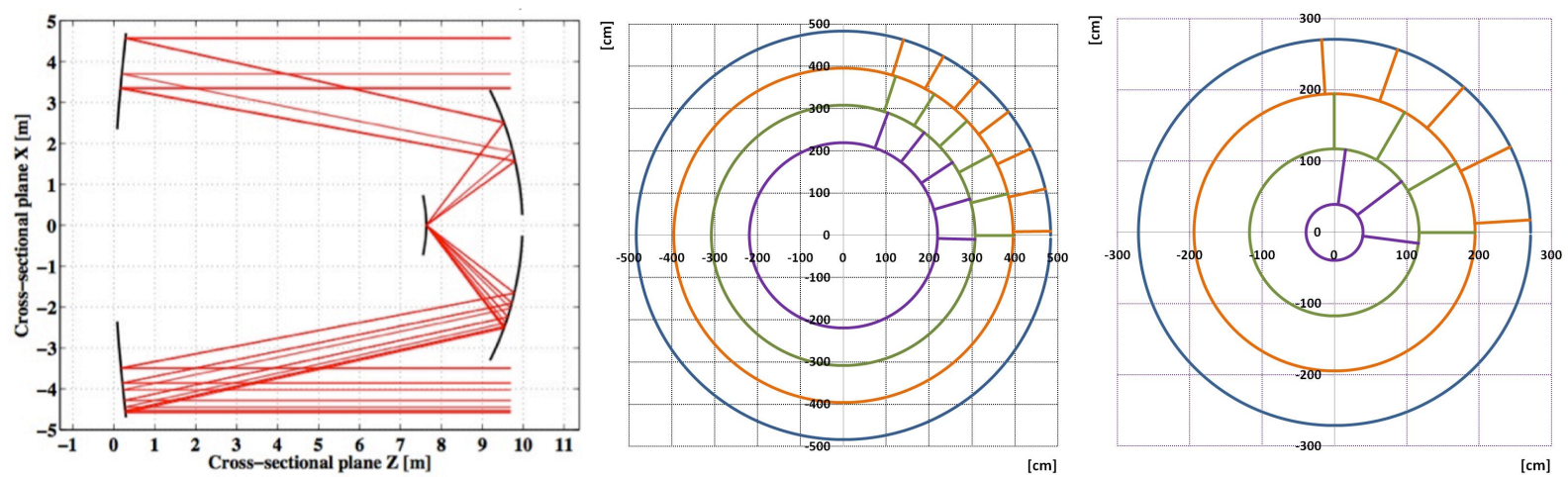

Figure 3. Left: The SCT optical geometry, showing example raytraces. Middle: The segmentation for the 3 segment rings of the primary mirror. Right: The segmentation for the 3 segment rings of the secondary mirror.

non-coherent light and operate a factor of $>1000$ above the diffraction limit. On the other hand, the required surface quality of the mirrors, of 2-5 nm RMS surface roughness on spatial scales equal to or smaller than the typical $330 \mathrm{~nm}$ wavelength of Cherenkov light (to prevent diffusive scattering from the mirror) is comparable to optical telescopes.

Extensive research of mirror replication technologies has identified those that are best capable of meeting the required specifications at competitive cost. In the mirror design and development planned for the production of the prototype SCT, differing mirror technologies will be used for the primary and secondary mirrors. We are collaborating with several potential industrial partners (Media Lario Technologies, MLT, in Italy, ATK Space Systems, Inc. and Electroformed Nickel Inc. in the US, and FLABEG in Germany) and have acquired sample mirrors or launched pilot research and development projects to assess the candidate technologies. Based on our assessment of the candidate technologies, the primary mirror panels will be manufactured by cold glass slumping, under the supervision of UCLA. For the secondary mirror for the prototype SCT, we plan to use electroformed mirror panels by electroforming, which will be manufactured under the supervision of collaboration members at the University of Huntsville in Alabama. Both the primary and the secondary mirror manufacturing methods require initial production of a high-quality aspheric mandrel (a mold or master), which is an expensive initial cost. The mandrels are then used to replicate multiple copies of aspheric panels to manufacture a ring of mirror panels, so that a cost benefit is realized for subsequent production.

In cold glass slumping, each panel is composed of two glass sheets joined by an aluminum Hexcel core. This technology is demonstrated on the MAGIC-II telescope and is currently the least expensive because the optical surface is derived from an optically flat glass sheet rather than from the moderately polished surface of the mandrel. The stress created in the glass sheet during mirror figuring is an important limitation, which makes this technology inapplicable for the secondary mirror, which has smaller radius of curvature. Electroforming for manufacturing high-precision replicated nickel mirrors with small radius of curvature has been demonstrated in a number of successful space-based, X-ray observatories (e.g. XMM-Newton) and is suitable for production of the secondary mirror. Because of the large initial cost of the mandrels for electroforming, we are planning to build only the single middle ring of panels for the secondary mirror of the prototype telescope, which is sufficient for telescope performance tests. Both sets of mirror panels will be coated with aluminum, and also given a protective over-coating by Bte Bedampfungstechnik GmbH in Germany or another vendor of similar capability.

The mechanical structure of the SCT will be sufficiently rigid that the mirrors will not need to be dynamically aligned in real time. However, seasonal variations in temperature will produce alignment changes that need to be corrected from time to time. The mirror alignment system, developed and prototyped at UCLA, will consist of a set of Hamamatsu position sensitive photo-detectors and corresponding small semiconductor lasers attached to the edges of the mirror panels, and a set of six low-cost linear actuators configured in a hexapod arrangement. The position sensors have been 
shown to provide relative displacement measurements of a few microns. The mirror alignment system will measure the panel-to-panel displacements, calculate the needed adjustments, and apply them to the actuators. The control system will consist of two computer systems, a small single board computer system (with controller for sensors and actuators) mounted on each panel and a fast central computer which will coordinate the panel computers and control large scale distortions of the optical system, such as focus, coma, and astigmatism, which will be derived from analysis of out-offocus images of moderately bright stars obtained with CCD cameras.

\section{TELESCOPE CAMERA}

The fine pixelation and wide field of view needed in the SCT focal plane camera, to match the capabilities of the SCT optical design and meet the science requirements of CTA, leads to a camera having about 11,000 pixels, each 0.067 degrees square. The small plate scale of the SCT design enables a highly-integrated camera using SiPM and custom ASIC electronics. Much of the data processing will be handled directly in the camera, which is therefore the core of the data acquisition system. The high degree of integration is key to realizing a low cost per channel in the camera, so that the fine pixelation enabled by the SCT design is affordable.

The camera structure is hierarchical, with the entire camera divided into a 9 subfields each covering a field-of-view of about 2.7 degrees, as shown in Figure 4. Each subfield is composed of 25 camera modules, and each of these consists of 64 detector pixels with front-end ASIC cards as shown in the figure. The division of the camera into subfields also provides a means of efficiently merging the signals from a large number of pixels into the front-end pattern trigger. For the prototype SCT, a camera containing only one subfield will be built initially. The subfield module normally will be deployed in the center of the camera, but can be relocated to other positions in the camera to evaluate off-axis performance.
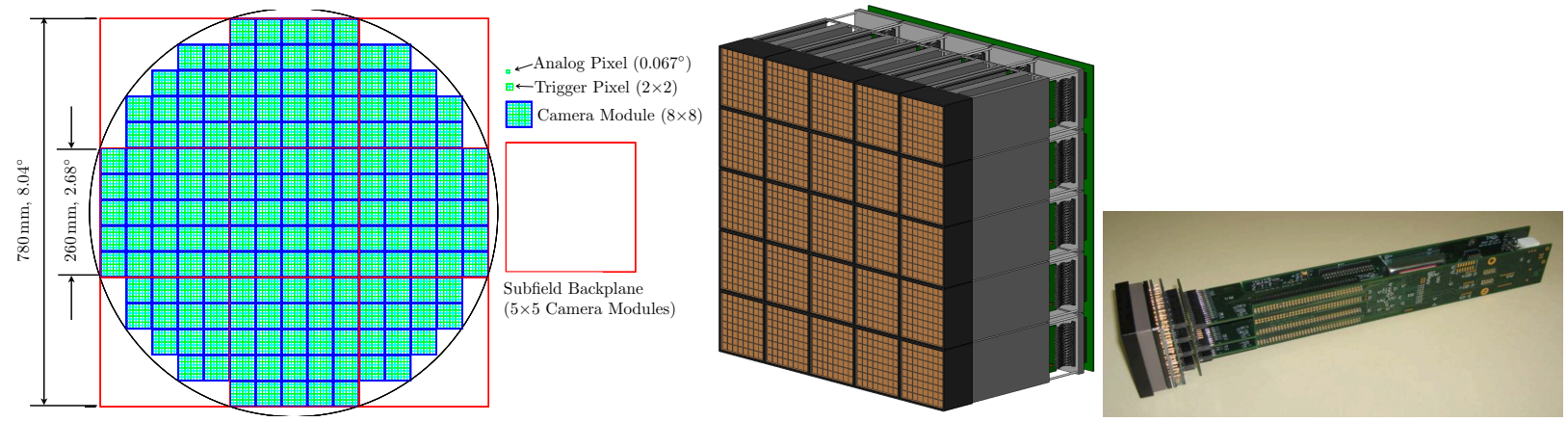

Figure 4. Schematic of the camera focal plane, showing division into 9 subfield backplanes each with 25 modules having 64 readout ("analog") pixels grouped into 16 trigger pixels. Middle: One camera subfield with 25 modules. Right: One camera module with front-end electronic boards and four TARGET ASICs. The module shown has a MAPMT, rather than a SiPM.

The focal plane (FP) sensors in the prototype camera will be "silicon photomultipliers" (SiPM), also known as Geiger avalanche photodiodes (GAPD) or multi-pixel photon counters (MPPC), which have greater photon detection efficiency (PDE) than vacuum photomultipliers. Additional advantages are operating voltage below $100 \mathrm{~V}$, little or no aging (even when exposed to bright light), and mechanical robustness. The baseline SiPM for the prototype camera is a Hamamatsu MPPC with $100 \mu \mathrm{m} \times 100 \mu \mathrm{m}$ cells, from the same series of detector elements used by the FACT Cherenkov telescope (http://www.isdc.unige.ch/fact/). It has a peak PDE of $27 \%$ at $400 \mathrm{~nm}$ and is available in arrays of $4 \times 4$ MPPCs, with each MPPC having an area of $3 \times 3 \mathrm{~mm}^{2}$. Four MPPCs will form one camera pixel and the 16 in an array will form one trigger pixel. Ongoing developments at Hamamatsu and other vendors, e.g. Excelitas, may soon offer improved alternatives, such as devices having higher efficiency in the UV. For example, some prototypes achieve more than 50\% PDE at 350 $\mathrm{nm}$. Collaboration members at UC-Santa Cruz, Georgia Tech, and Barnard College will work on the testing, procurement, and calibration of the SiPM and their integration into the prototype camera.

A complication of using SiPMs is a relatively strong temperature dependence of gain (typically $1 \%$ per $1 \mathrm{C}$ ), dark rate, and PDE, which could potentially lead to large systematic uncertainties in the SCT response. These uncertainties are 
minimized if the gain and PDE do not change by more than $\sim 1 \%$, which is accomplished by enclosing the FP in an environment with the temperature stabilized to better than $0.5 \mathrm{C}$. The thermally insulated FP enclosure has a UV transparent entrance window and insulated sidewalls. The camera modules fit together in such a way as to form a thermal barrier between the focal plane and electronics volumes in the camera. The main source of FP heating, the readout electronics, is sufficiently small that the SiPM can be effectively cooled to 0-20 C by thermoelectric elements. The warm sides of the thermoelectric elements are on the electronics side of the barrier and are cooled together with the electronics. Condensation within the box is avoided using silica gel cartridges and dry nitrogen flushes. The front-end electronics for each module consists of a stack of boards with four 16-channel analog pipeline TARGET ASICs, each with a readout FPGA (Figure 4). The specifications are shown in Table 1.

Table 1. Camera front-end electronics specifications.

\begin{tabular}{|l|l|}
\hline Sampling rate & 1 Gsample per second \\
\hline Memory depth & 16 microseconds \\
\hline Dynamic range & 9 bits \\
\hline Analog bandwidth & $500 \mathrm{MHz}$ \\
\hline Maximum readout rate & $10 \mathrm{kHz}$ random \\
\hline Single pixel trigger rate & $200 \mathrm{MHz}$ \\
\hline Single telescope trigger rate & $10 \mathrm{MHz}$ accidental \\
\hline Electronic cross talk & $\sim 40 \mathrm{~dB}$ maximum \\
\hline
\end{tabular}

The TARGET ASIC design follows from more than 6 generations of the LABRADOR and BLAB ASICs designed by a group at the University of Hawaii led by G. Varner. An initial version of the TARGET chip and electronics for the SCT camera has been extensively tested at the SLAC National Accelerator Laboratory ${ }^{5}$. Each electronics module forms a discriminated analog sum of each of the 16 groups of 4 adjacent pixels and delivers these 16 trigger signals to the backplane connector. Each module has programmable SiPM voltage supplies and discriminator thresholds and an ADC for monitoring currents. Other outputs to the backplane include a high-speed serial data stream, a slow control serial link (for setting discriminators and supply voltages and reading out currents), and several high-speed handshaking signals.

The electronics modules plug directly into the camera subfield backplane, which has the mechanical and electrical interfaces for 25 64-pixel electronics modules, plus a means for positioning modules into a curved focal plane, as needed for the SCT camera. The backplane has a fast low-level pattern trigger using a single FPGA to identify clusters of hits, and data acquisition hardware to collect the serial data streams from the ASIC boards and transmit them through a highspeed interface to data-acquisition (DAQ) computers. A prototype of this DAQ scheme has been tested at Washington University in St. Louis (WashU), and an 8-lane PCIe Version 2 system should handle burst rates up to 4GB/s.

The camera mechanical system design, led by collaboration members at the University of Chicago, consists of the chassis which houses the camera electronics and includes a camera cooling system, the interface to the OSS, a mechanical frame for supporting camera backplanes and the focal plane, and a Stewart platform for optical alignment of camera focal plane. Ancillary systems for the camera mechanical system include environmental monitoring and an automatic shutter system. The camera design will allow for in-field replacement of camera subfields or modules. The optimum use of the SCT in conjunction with VERITAS requires an array trigger that can assemble information from all the telescopes and distribute the appropriate trigger signals. Groups at Iowa State University and ANL will implement the needed trigger system for the prototype SCT and VERITAS, based on their recent upgrade of the VERITAS trigger.

\section{TELESCOPE TESTING}

We plan to test the SCT by co-locating it with the existing VERITAS array of four 12-m IACTs at the Fred L. Whipple Observatory in Arizona, operated by the Smithsonian Astrophysical Observatory. The observatory has the experienced staff and infrastructure to erect and operate the SCT. An existing vacant telescope pier, previously used by a VERITAS telescope, can readily host the SCT. Electrical and telecommunications wiring near the proposed site already exists, as 
do trailers and buildings for hosting the electronics and control systems. SAO will coordinate work to host the telescope, including ensuring a match between the existing pier sole plate and the telescope, plus trenching and cabling as needed for power and data connections to the SCT, and proving a trailer for the hosting the telescope electronics. SAO also will provide an onsite staff member to coordinate the installation and locally lead the site testing and debugging.

\section{SUMMARY}

Proposals have been submitted to the NSF and Department of Energy in the US for the development, fabrication, and commissioning of a prototype Schwarzschild-Couder telescope for use with the Cherenkov Telescope Array. The prototype telescope could be accomplished in 3 years, which would allow the US to move on to contributing 36 SC telescopes to CTA on a schedule compatible with the existing CTA schedule. The SCT tasks would be performed by a consortium consisting of 10 universities plus SAO and the Argonne and SLAC National Laboratories in the U.S., with international partners at DESY-Zeuthen in Germany and IFJ PAN in Krakow, Poland. The U.S. institutions are leaders in the fields of ground-based and space-based gamma-ray astrophysics with a record of successfully delivered scientific instrumentation for the Fermi, VERITAS, and Milagro projects, and have the expertise and capabilities needed to accomplish this project. The DESY-Zeuthen and IFJ PAN- Krakow groups can provide non-US-funded engineering support for the development of the SCT mechanical design, making use of their existing design and development work for the medium-sized and small-sized telescopes for CTA. Figure 5 shows the possible schedule for the program, which is divided into 3 main consecutive phases: a 1-year design phase from August 2012 to August 2013, followed by a 13month construction phase ending in September 2014, followed by an 11-month commissioning phase ending in July 2015. Finally, the schedule shows the transition to an on-going Operations Phase starting in August 2015.

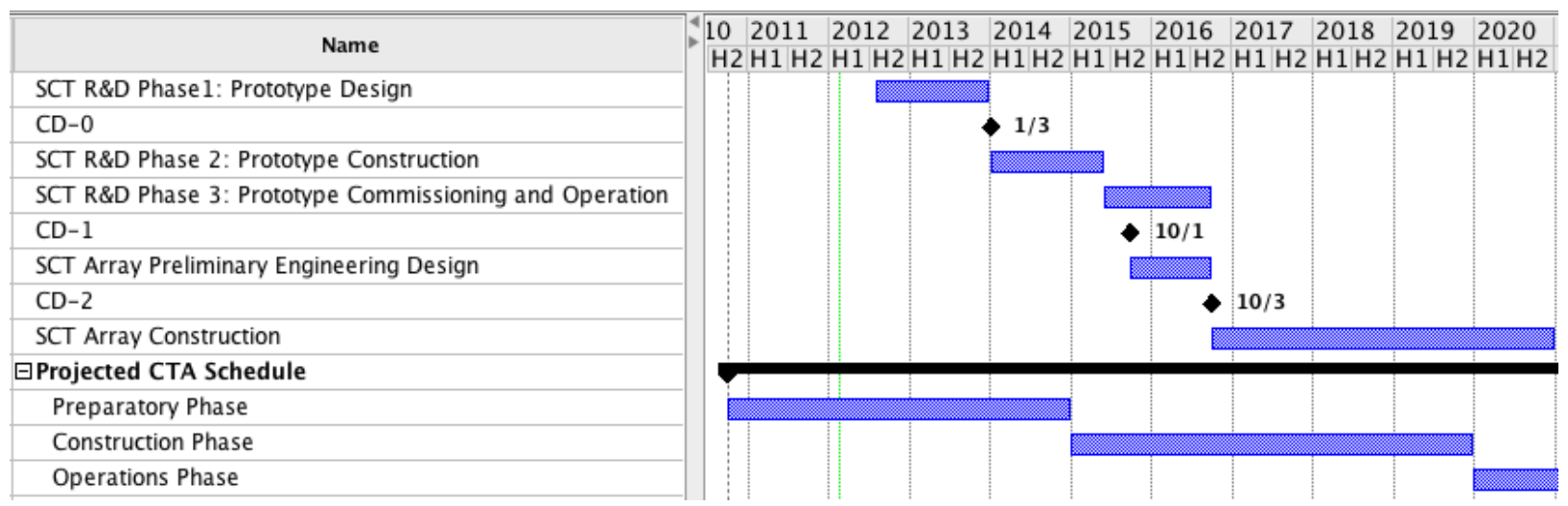

Figure 5. An candidate top level schedule for the design, construction and use of a prototype SCT, with representative dates for three Critical Decision milestones for such a project. Also shown are the projected Preparatory, Construction and Operations Phases for the main CTA schedule.

\section{REFERENCES}

[1] Pareschi, G., "The Cherenkov Telescope Array (CTA): status of the project and development of the telescopes", Proc. SPIE 8444 (2012).

[2] Vassiliev, V., Fegan, S. and Brousseau, P., "Wide field aplanatic two-mirror telescopes for ground-based $\gamma$-ray astronomy", Astroparticle Physics, 28,10-27; arXiv:astro-ph/0612718 (2007).

[3] Vassiliev, V. V. and Fegan, S. J., "Schwarzschild-Couder two-mirror telescope for ground- based gamma-ray astronomy", International Cosmic Ray Conference, 3,1445-1448; arXiv:0708.2741 (2008).

[4] Schwarzschild, K., "Undersuchungen zur geometrischen Optik II", Astronomische Mitteilungen der UniversitaetsSternwarte zu Goettingen. 10,1-28 (1905).

[5] Bechtol, K., Funk, S., Okumura, A., Ruckman, L. L., Simons, A., Tajima, H., Vandenbroucke, J. and Varner, G. S. "TARGET: A multi-channel digitizer chip for very-high-energy gamma-ray telescopes", arXiv:1105.1832 (2011). 